Extended abstract

\title{
Microbial interactions in the rumen
}

\author{
RA Prins ${ }^{1}$, CS Stewart ${ }^{2}$ \\ ${ }^{\prime}$ Microbial Ecology Group, CEES, University of Groningen, Kerklaan 30, 975I NN Haren, \\ The Netherlands; \\ ${ }^{2}$ Rowett Research Institute, Bucksburn, Aberdeen AB21 9SB, Scotland, UK
}

Since the publication of "The Rumen and its Microbes" by R.E. Hungate in 1966 [1] - a landmark in rumen microbiology - many developments have taken place in this field. Some of the progress will be reviewed, if only to show that various ideas originally formulated by Hungate, e.g. the concept of the turnover of extracellular fermentation intermediates (hydrogen, succinate, formate) in the rumen, the drive for 'maximum biochemical work', and the prediction of a preponderance of microbial and enzymatic synergisms, were indeed confirmed. These predictions have to be viewed in the light of the 1960 's when coexistence of microbial species and cooperative interactions were not often stressed. Instead, it became fashionable to study competition experiments in chemostats. At present, rumen microbiology enjoys another boom period, mostly as a result of the development of improved techniques from molecular biology. While the culturability of rumen bacteria and fungi is good in comparison with the situation encountered for oligotrophic habitats (sea, soil, groundwater), it is now also possible to trace the fate of organisms in the numen by in situ detection, to record the specificity of protozoal predation on bacteria and to unravel the true diversity beyond or within the species level. This is important, as many old questions remain as yet unanswered.

\section{Microbial diversity}

Much of the biochemical and microbial diversity that evolved in the rumen can be explained on the basis of the complex and variable composition of plant matter, the physico-chemical heterogeneity at the microscale and the continuously-occurring fluctuations in feed intake, salivation, oxygen exposure and other parameters. But even when conditions remain constant a considerable genetic heterogeneity can be expected to develop among and within species [2]. Training rumen bacteria by growth on specific fibre sources leads to the emergence of mutant strains with greatly enhanced fibrolytic activities able to out-compete the starting strains [3]. Comparable events in the rumen were presumably involved in the emergence of the present-day rumen strains. The molecular events involved in these changes are not yet fully understood. 
Among the hundreds of bacterial species in the rumen only some thirty or forty form the bulk of the bacterial biomass, carrying out the major part of microbial digestion. Unexpectedly, minority species may hold key functions, as has been demonstrated for the fermentation of proteins, mucins and toxic plant metabolites. Much of the evolution of enzymes in heterotrophic microorganisms is centered around polymer cleavage and monomer transport. The peripheral pathways of biopolymer cleavage are more diverse than the ensuing fermentation mechanisms into which breakdown products are fed, as almost all fermentative polymer utilizers have the same glycolytic pathway for the processing of the monomers. For every microbial function - e.g. the fermentative breakdown of cellulose - there are several microbial candidates, but at present it seems that no two are identical.

\section{Polymer hydrolysis}

New insights into the nature of the surfaceassociated cellulase complexes of numen organisms are reviewed by Flint and by Forano in this publication. Such cell-surface complexes are unique to anaerobes [4]. In contrast, Salyers and her colleagues have shown that the amylose, amylopectin and pullulan degrader Bacteroides thetaiotaomicron possesses polymer-binding complexes, whilst polymer-hydrolysing enzymes await in the periplasmic space together with proteins that bind to the oligosaccharide products which are subsequently transported into the cell and cleaved to monosaccharides [5]. Thus different strategies exist with more or less spilling of cleavage products and therefore more or less cooperation with non-polymerutilizing species. In vivo, these strategies must have consequences for other organisms and must have a meaning in terms of survival value of hosts, and not only on the availability of food during different feeding situations. From the work of Flint. Wood and others it has become clear that we deal with a considerable number of synergistic mechanisms for the anaerobic breakdown of cell wall polysaccharides. Some microbial xylanases and cellulases show intramolecular synergisms between binding domains, catalytic domains for the cleavage of the backbone and domains for cleaving side-chains. Other examples of cooperative action are found between different hydrolytic enzymes from polymerutilizing organisms. Then there are synergisms between primary polymer-degraders and secondary utilizers of oligomers. A fierce competition for the liberated monomers and dimers results in the rapid uptake by non-muralytic bacteria and protozoa preventing feedback inhibition of the initial cleavage process [6].

Cross-feeding of nutrients and competition for substrates are not the only regulatory chemical interactions between microorganisms in the rumen. Differential toxicity of nutrients in the feed could be more important in gut systems than is currently thought. Plant metabolites in extracts from the tropical browse species Calliandra supress the growth of Ruminococcus and $\mathrm{Fi}$ brobacter strains, but have less effect on strains of Butyrivibrio and Selenomonas [Salawu et al., unpublished]. Similar extracts from leaves of Acacia sp. inhibit the growth of selected Gram positive, but not Gram negative, rumen bacterial strains. Such effects are probably commonplace in the rumen of animals fed leguminous forage or browses, which contain a variety of toxic metabolites.

An increasing number of antagonisms and examples of chemical warfare are being described. Several species of physiologicallydifferent rumen bacteria including cellulolytic 
and chitinolytic species inhibit cellulolytic rumen fungi; the enzymic processes involved probably have some important common features [7, Stewart et al, unpublished]. The outcome of competition between various cellulolytic numen bacteria, especially Ruminococcus species, depends not only on the substrate offered but in addition seems to be regulated by the formation of antagonistic compounds, probably bacteriocins [8]. How the production of bacteriocins favours invasion by cellulolytic strains of Ruminococcus remains to be investigated. The best studied example of the evolution and ecological role of bacteriocins is found in Escherichia coli, a gut bacterium normally present in only low numbers in the rumen, but now of considerable interest because of the extreme toxicity to humans of some strains that can spread from sheep and cattle. In colicinogenic $E$. coli, genes encoding production, transport and immunity occur as tightly linked clusters on plasmids. According to the "diversifying selection" hypothesis of Riley, positive selection favours new strains bearing mutations in the immunity genes or in the immunity binding domain of the colicin genes and results in the establishment of those strains with novel immunity functions as a result of the colicin killing effect of the new immune strains [9].

\section{Fermentation intermediates}

The concept of extracellular rumen proved to be valid and probably holds for gastrointestinal fermentations intermediates in other herbivores. The importance of dihydrogen, formate, succinate and the minor role played by ethanol and lactate have been confirmed. Lactate, however, still is an intermediate of some significance during sudden high rates of hexose flux. The measurement of the two rivaling randomising and acrylate pathways for the fermentation of lactate is a textbook example of the use of label in microbial ecology, yet it is not known whether rumen microbes other than Megasphaera elsdenii possess the acrylate pathway. The acrylate pathway seems to be more prevalent in cattle than in sheep, but this has not been explained in terms of differences in the microbial populations.

The generation of a sodium- or protonmotive force during the decarboxylation of organic acids (oxalate, succinate) is one of the exciting new concepts in the microbiology of gut anaerobes. These reactions are carried out by specialist strains (Oxalobacter formigenes, Succiniclasticum ruminis) and in view of the many low-energy organic acids occurring, for example, in herbage, the isolation of additional specialists can be expected.

\section{Anaerobic respirations}

In nature, fermentations are often coupled to anaerobic respirations, provided that the extracellular electron acceptors are continuously supplied or regenerated as is the case at oxic-anoxic interfaces in sediments and soils. The rumen is characterized by a scarcity of such electron acceptors: the only important extracellular electron acceptor seems to be bicarbonate, itself a fermentation product.

Nitrate, sulfate and oxidized forms of iron are only present in trace amounts in normal feeds, and they are not generated in the rumen. The possible importance of other electron acceptors has received little attention: the reductive removal of side chains from aromatic compounds, the possible role of polysulfides as electron-shuttles or the role of iron-reducing bacteria using polyphenols and quinones as electron-shuttles towards oxygen have not been studied in a quantita 
tive way. Mass spectrograms of rumen fluid show the presence of a multitude of aromatic compounds, but their metabolism is not very well known.

Even in methanogenic systems such as the rumen, acetogens are found in high numbers. The question remains whether these exist as fermentative organisms only, transferring the hydrogen to methanogens, or whether autotrophic acetogenesis takes place in certain circumstances, which remain to be elucidated.

1. Hungate RE (1966) The Rumen and its Microbes. Academic Press, London and New York .

2. Lenski RE (1995) Mol Ecol 4, 643-65 1
3. Saluzzi L (1993) Ecophysiology of Cellulolytic Bacteria in the Rumen. PhD thesis, University of Aberdeen

4. Warren RAJ (1996) ASM-News 62, 85-88

5. Anderson KL, Salyers AA (1989) $J$ Bacteriol 171, 3192-3198

6. Flint HJ, Forsberg C. (1995) In: Ruminant Physiology: Digestion, Metabolism, Growth and Reproduction, (Engelhardt WV, Leonhard-Morek S, Breves $G$ and Gieseke D eds), Ferdinand Enke, Verlag, Stuttgart, 43-70

7. Kopecny J, Hodrova B, Stewart CS (1996) Lett App Microbiol 23, 199-202

8. Odenyo AA, Mackie RJ, Stahl DA, White BA (1994) Appl Environ Microbiol 60, 3688-3696

9. Tan Y, Riley MA (1996) Microbiol 142, 2175-2180 